\title{
Technical Note: A Method to Facilitate Retrieval of Polyester Bags Used in In Sacco Trials in Ruminants
}

\author{
C. W. Cruywagen \\ Department of Animal Sciences, Stellenbosch University, Private Bag X1, Matieland, Stellenbosch 7602, South Africa
}

\begin{abstract}
A method is described to facilitate the retrieval of polyester bags from ruminally cannulated cattle. The system consists of 2 major components, i) an accommodation vessel for multiple polyester bags in the form of an opaque nylon stocking, referred to as the "receptacle," and ii) a shorter stocking, knotted with a loop on one end to attach to the receptacle, referred to as the "catcher." The catcher is tied on the other end to the inside of the cannula plug. Sample bags are inserted in tandem into the receptacle, separated from each other by a tight knot. The receptacle is tied to the catcher and subsequently inserted into the rumen. Upon retrieval, receptacles are pulled out only far enough to expose the first bag to be removed. This procedure ensures easy bag retrieval without exposing the remaining bags (intended for further incubation) to air.
\end{abstract} Key words: in sacco trial, degradability, experimental method

The experimental procedure for in sacco trials with ruminally cannulated animals is well documented. Methods described by the Agricultural and Food Research Council (1992) and the National Research Council (2001) are widely accepted and applied for the determination of nutrient (especially protein) degradability. These methods are quite specific regarding sample preparation, chemical analyses required, bag specifications, number of replications, incubation times, and mathematical models to be used for data analyses. However, there does not appear to be a standard recommended procedure pertaining to the way in which bags are accommodated in the rumen. Different laboratories use different methods to incubate bags in the rumen; for example, individually weighted bags attached to nylon strings, bags placed into nylon laundry bags, or bags attached to stainless steel discs to retain them in the rumen. In all of these procedures, bags often get tangled or bunched, which may either complicate re-

Received September 2, 2005.

Accepted October 21, 2005.

E-mail: cwc@sun.ac.za trieval or affect microbial access to the substrate being incubated. To retrieve time-specific bags, most procedures result in transient removal of bags intended for further incubation, thus exposing them to air, which could affect subsequent microbial degradation. The exception is the situation in which all the bags are removed simultaneously. Although simultaneous removal of bags has been used in many studies and has been a recommended procedure for some time (Nocek, 1988), simultaneous insertion should be the preferred option because it would ensure similar conditions for microbial attachment to the substrate (Vanzant et al., 1998).

This note presents a method for polyester bag accommodation in the rumen that facilitates bag retrieval and would prevent unnecessary exposure to air of bags intended for later removal. The method can be applied in any ruminant animal equipped with a rumen cannula that is suitable for in sacco work. Although measurements provided in this note apply to cattle, the method could be adapted for use in small ruminants as well.

The system consists of 2 major components, i) an accommodation vessel for multiple polyester bags in the form of an opaque nylon stocking, referred to as the "receptacle", and ii) a shorter stocking, knotted with a loop at one end to attach to the receptacle, referred to as the "catcher". The catcher is made by cutting a 300$\mathrm{mm}$ section from one leg of a lady's stocking. One end is looped backwards and tied to itself to form a loop about $100 \mathrm{~mm}$ long. The other end is tied to the inside of a cannula plug. The receptacle is made from the entire length of one leg of an extra large opaque stocking. Opaque stockings are used for the receptacles because of their strength. Cameo winter opaques (Burhose, Brackenfell Industria, Cape Town, South Africa), knitted with 44 decitex yarn and 17 decitex Lycra, are used in our laboratory. The mesh size was determined with the aid of a Nikon Eclipse E400 microscope (Nikon Instruments, Kanagawa, Japan) set at $40 \times$ enlargement and the Simplex PCI version 4.0 computer program (Compix Inc., Imaging Systems, Tualatin, OR). Mesh diameter of the material was found to average $151 \pm 74 \mu \mathrm{m}$ unstretched, and $227 \pm 67 \mu \mathrm{m}$ when 


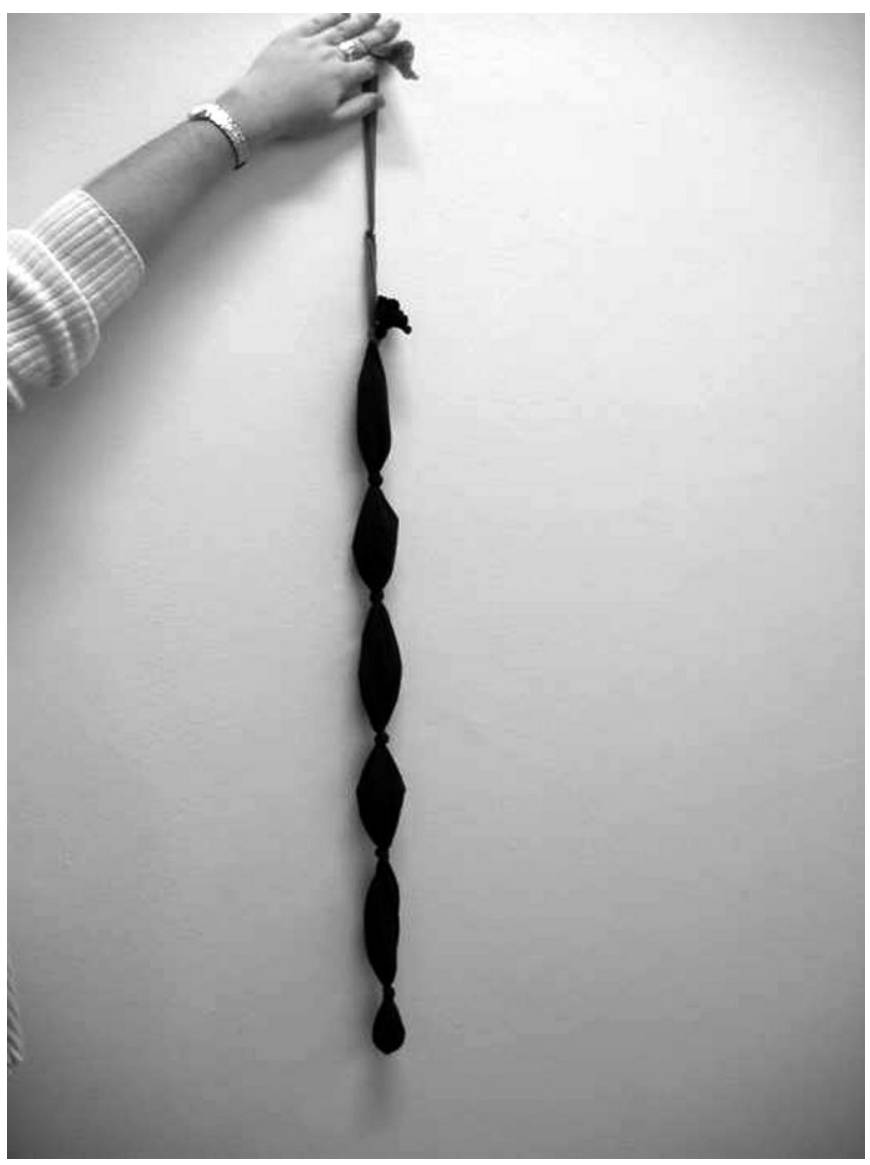

Figure 1. Catcher and receptacle containing bags for ruminal incubation. A marble is tied in the toe end to act as weight.

stretched. It therefore appears that mesh size would not limit passage of rumen liquor through the receptacle to the polyester sample bags contained inside the receptacle. It is also expected that the weight of the marble and the movement of the rumen contents would result in the material being stretched frequently and that this would further enhance the passage of rumen liquor through the material.

One large glass marble (ca. $33 \mathrm{~mm}$ in diameter, weighing ca. $50 \mathrm{~g}$ ) is used as a weight and is inserted into the toe end of the receptacle stocking (Figure 1). A tight knot is made just behind the marble to keep it in position. The polyester bags containing the feed samples, appropriately identified and sealed, are then inserted in tandem into the receptacle, separated from each other by a tight knot. Blank bags should be placed as required. The distance between knots should be at least $100 \mathrm{~mm}$. Depending on the length of the opaque stocking, about 5 polyester bags could be inserted in each receptacle (Figure 1). Before inserting the bags, the receptacle is stretched out to approximately $1.8 \mathrm{~m}$ and relaxed again; this increases the overall length and

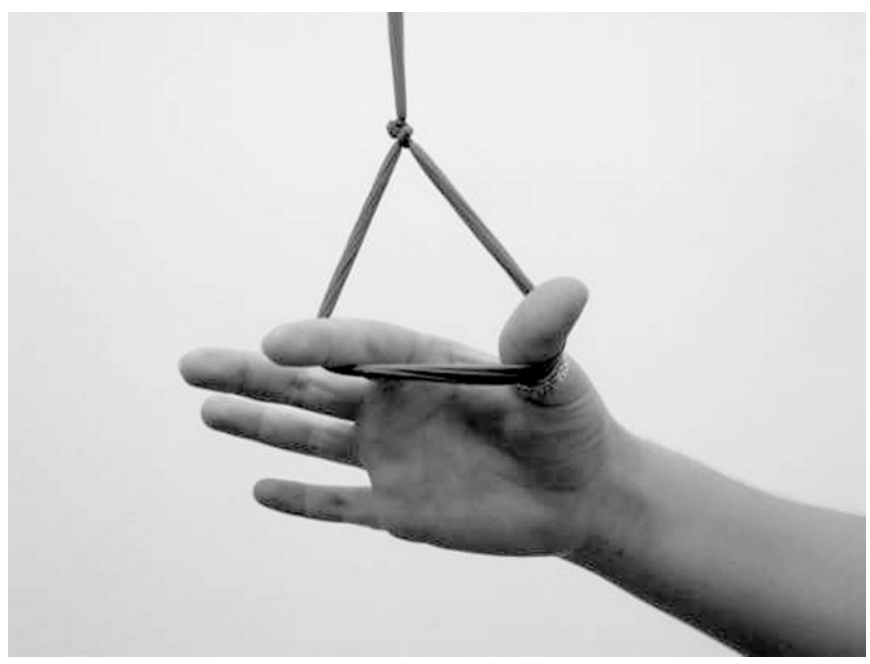

Figure 2. A sling is formed in the loop of the catcher to attach to the receptacle.

ensures sufficient space for 5 bags. Each receptacle is then attached to its catcher in the following way: By pushing the thumb and index finger through the loop, a piece of the catcher is pulled back through the loop to form a double-stranded sling (Figures 2 and 3), which is then placed beneath the knot above the last bag inserted into the receptacle, and pulled tight (Figure 3). Note: The loop in the catcher should be long enough (at least $100 \mathrm{~mm}$ ) to ensure that the knot tied into the catcher itself to form the loop does not get entrapped in the sling. Catchers can be coded (to identify treatments or substrates) by tying different colored elastic bands around them in the area where they are fastened to the cannula plug.

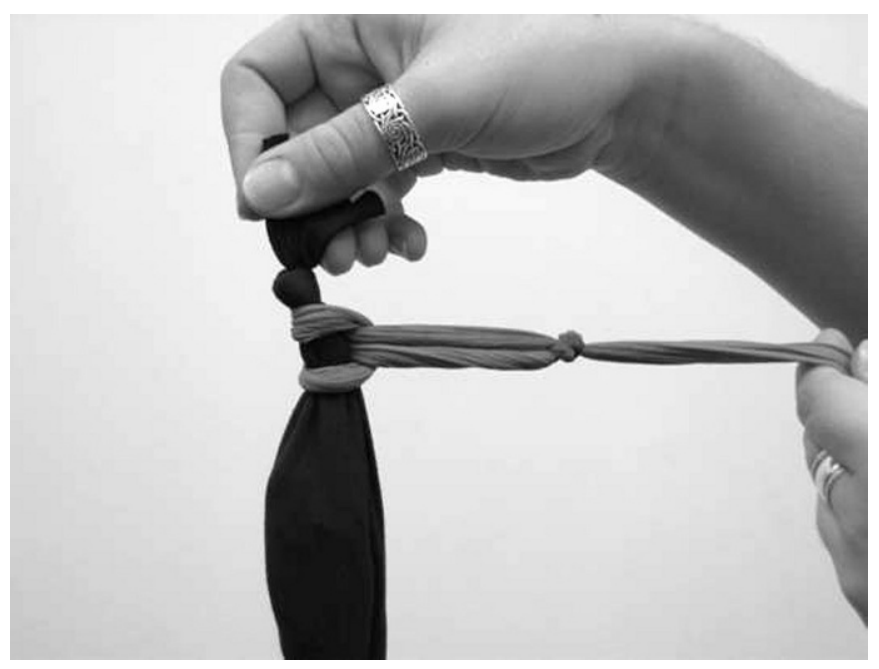

Figure 3. The sling is tied to the receptacle. 


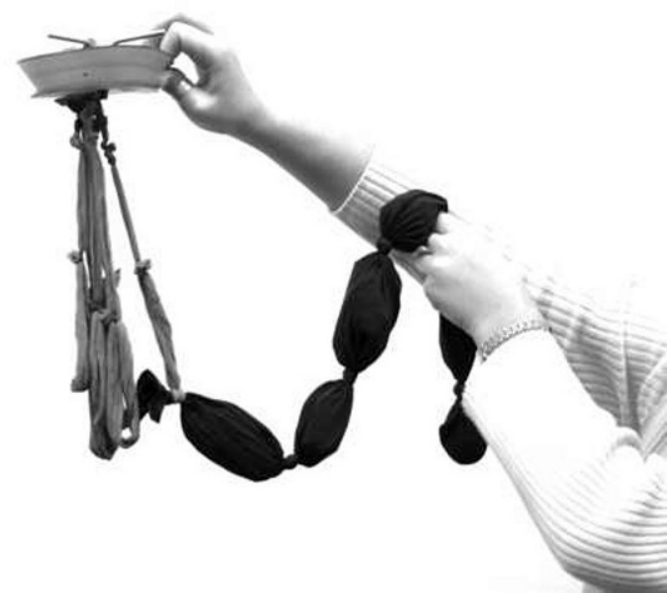

Figure 4. Receptacle containing polyester bags, tied to the catcher and cannula, and ready for rumen incubation.

After all the receptacles have been securely tied to their catchers (Figure 4), they are inserted into the rumen and the cannula plug is replaced. The catcher is long and stretchy enough for the receptacle to be pulled deep into the rumen, thus covering the bags for the duration of the incubation period. For cattle, no more than 6 full-length receptacles should be inserted into the rumen at one time.

At each bag removal time, the following procedure is followed: Remove the cannula plug and pull catchers out far enough only to expose the segment of the receptacle that is tied to the catcher. With a pair of scissors, carefully cut the (first) knot just above or just below the catcher sling (Figure 5) and pull the receptacle free from the sling. The loop in the catcher can then be reopened again easily. (If the receptacle is cut below the sling, care should be taken not to cut the polyester bag in the process; work the bag away from the cutting area). Pull the first sample bag to be retrieved from the receptacle. Next, cut the receptacle just above the second knot to remove the empty segment that contained the retrieved bag. The next segment of the receptacle becomes the following one to be tied to the catcher

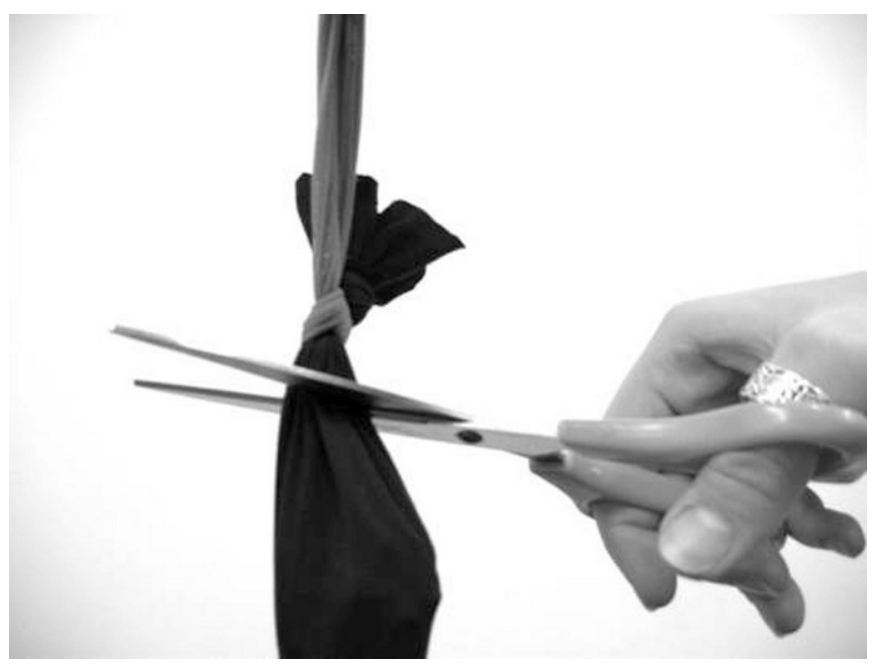

Figure 5. During bag retrieval, the receptacle is freed from the catcher by cutting just above or just below the sling.

loop. Repeat the process for each receptacle segment that has to be removed, push catchers back into the rumen, and replace the cannula plug.

If blank bags are used in an in sacco trial, they can either be incubated in a separate receptacle and removed at designated times, or distributed among sample bags. In the latter case, 2 receptacle compartments (blank bag and sample bag) would be removed each time that a blank bag has to be retrieved. This procedure has been used successfully in our laboratory in a series of trials and has replaced previous incubation methods.

\section{REFERENCES}

Agricultural and Food Research Council. 1992. Nutritive requirements of ruminant animals: Protein. Report No. 9 of the Technical Committee on Responses to Nutrients. Nutr. Abstr. Rev. B 62:787-835.

National Research Council. 2001. Nutrient Requirements of Dairy Cattle. 7th Rev. ed. Natl. Acad. Sci., Washington, DC.

Nocek, J. E. 1988. In situ and other methods to estimate ruminal protein and energy digestibility: A review. J. Dairy Sci. 71:2051-2069.

Vanzant, E. S., R. C. Cochran, and E. C. Titgemeyer. 1998. Standardization of in situ techniques for ruminant feedstuff evaluation. J. Anim. Sci. 76:2717-2729. 